\title{
Association between depressive symptoms and medical care utilization in South Korean older adults with chronic diseases: A cross-sectional study
}

\author{
Yoon Sik Park \\ Yonsei University College of Medicine \\ Eun-Cheol Park ( $\nabla$ ecpark@yuhs.ac) \\ Yonsei University College of Medicine
}

\section{Research Article}

Keywords: Depressive symptoms, medical care utilization, chronic disease, public health, elderly, South Korea

Posted Date: August 11th, 2021

DOI: https://doi.org/10.21203/rs.3.rs-676687/v1

License: (1) (1) This work is licensed under a Creative Commons Attribution 4.0 International License.

Read Full License 


\section{Abstract}

\section{Background}

This study examined the effects of depressive symptoms on medical care utilization among South Korean older adults with chronic diseases.

\section{Methods}

A national sample of 3,921 elders with chronic diseases participated in the Korean Longitudinal Study of Aging. Using multiple logistic regression, odds ratios (ORs) were calculated for the effects of depressive symptoms on medical care utilization.

Results

Older adults with depressive symptoms had fewer outpatient visits (OR $=0.81,95 \%$ confidence interval $[\mathrm{Cl}]=0.67-0.98)$ and more inpatient hospitalizations $(\mathrm{OR}=1.49,95 \% \mathrm{Cl}=1.13-1.97)$ than those who did not suffer depressive symptoms. Participants with depressive symptoms visited outpatient clinics less often $(\mathrm{OR}=0.69,95 \% \mathrm{Cl}=0.48-0.97)$ and were hospitalized more frequently $(\mathrm{OR}=1.81,95 \% \mathrm{Cl}=1.04-$ 3.16) than were men without depressive symptoms. Patients with depressive symptoms with hypertension and diabetes were hospitalized more often $(\mathrm{OR}=2.77,95 \% \mathrm{Cl}=1.45-5.30)$.

\section{Conclusions}

Depressive symptoms and chronic diseases are common among elders in South Korea, often resulting in decreased outpatient visits and increased inpatient hospitalization. These findings suggest the necessity for management of chronic disease in elders with depressive symptoms.

\section{Background}

Depression is a major public health concern that affects about 279 million people worldwide. It is the one of leading problems of the growth in the global burden of diseases [1]. Depression is commonly spread among elders and negatively effects on health and quality of life in the rest of life [2,3]. Among all members of the Organization for Economic Co-operation and Development (OECD), South Korea showed second place of the highest rates of suicide committed among elders. Therefore, depression of elders in South Korean population has took serious concern as a national health topic recently [1]. Several studies have been established on depression among elders in South Korean population so far, but a lot of researches have concentrated on the factors associated with depression itself not on health management. Usually, depression could be medically diagnosed and managed but is sometimes underestimated and remains without cares. The inappropriate management of depression is one of the well-known risk factors for suicide, an increasingly serious public health problem. [2]. 
Although many former researches have tested the relationship between depressive symptoms and health, few have founded the effect of depressive symptoms on medical care utilization among elders in South Korean population $[4,5]$. Thus, interest in the utilization of medical care by older adults with depressive symptoms is arising, depressive symptoms are often misdiagnosed or untreated in this group of population, which may make their use of medical care services lower than that by patients who are not suffering from depression $[2,6]$. However, there is no consensus on the impact of depressive symptoms on health care service utilization in older adults. Some studies have found that depressive symptoms are positively related to healthcare utilization, but others have found mixed or no such associations $[7,8]$. There may be confounding factors associated with coexisting chronic diseases.

In 2017, the prevalence of diagnosed hypertension and diabetes in South Korea was approximately 59.0\% and $23.2 \%$, respectively, which has been increasing over the years [9]. Former studies researching the associations between suffering depressions and medical care utilization showed increased usage of medical care service among elders with chronic disease such as hypertension, however having chronic diseases are often merely regarded as independent variables $[10,11]$. Some of researches have studied the complex associations of chronic disease and depressive symptoms with medical care utilization and have described a relationship between coexisting conditions and high rates of health care utilization [12,13]. Moreover, these studies were not conducted in South Korea. Therefore, there is not much known about whether such association exists among elders in Asia, especially South Korea. South Korea is the most drastically aged country of the world. Even though South Korea has national insurance service with universal coverage named the National Health Insurance (NHI), high rate of out-of-pocket payments still have been an issue because of the benefits are limited. The payment system of $\mathrm{NHI}$ is based on fee-for-service payment, resulting in increased usage of medical care service, and disadvantages for executing coordinated or managed medical care for patients with combined health status, such as coexisting depressive symptoms and chronic diseases together [14].

Medical care utilization is defined as the outcome of the relationship between medicine professionals and patients [15]. It can be a good indicator of the national healthcare system. The measures associated with utilization are often a function of the volume of services and costs [16]. The purpose of this study was to find the relationship between suffering depressive symptoms and medical care utilization. Furthermore, we aimed to discover the combined relationship of suffering depressive symptoms and chronic diseases with medical care utilization among elders in South Korean population [17].

\section{Methods}

Data

Used data for this paper was acquired from the Korean Longitudinal Study of Aging (KLoSA). Similar to same kind of studies such as the Survey of Health, Aging, and Retirement (SHAR) in Europe and the Health and Retirement Study (HRS) in the United States, KLoSA provides data collected from interviews on various perspectives of the latter years of adults in South Korea, including medical care facility 
utilization, relationships between family members, income, employment and retirement of job. Although, KLoSA is a longitudinal study of adults aged over 45 years old at the baseline of study in 2006, this study analyzed the 2018 interview data of 3,921 people aged over 55 years at the 2018 survey.

\section{Variables and measures}

Medical care utilization

Medical care utilization was the dependent variable in this study. It was categorized into two groups: inpatient hospitalization and outpatient visits. Inpatient hospitalization was defined as a person hospitalized at least once in the past two years. Outpatient visits were defined as at least 12 visits (once every 2 months) within the last two years. In South Korea, patients with chronic diseases are usually recommended visiting an outpatient clinic once a month. Therefore, if a patient visited an outpatient clinic at least once every two months, they were considered to meet the criterion for outpatient visits.

\section{Depressive symptoms}

The interesting variables of this study were depressive symptoms. We used 10-item Centre for Epidemiological Studies Depression Scale (CES-D-10) questionnaire to measure depressive symptoms. The CES-D-10 is a 10-item questionnaire, which evaluates depressive symptoms suffered in the past week. It contains three questions on depressive mood like feeling depressed, five questions on somatic symptoms like feeling pain, and two questions for positive affect. There are four choices for each questions from score 0 ("rarely or none of the time") to score 3 ("all of the time"). Scoring is reversed for question number 5 and 8 ("rarely of none of the time" is score 3 and "all of the time" is score 0 ), which are positive questionnaires. With total scores ranging 0 to 30 , the cutoff value for depressive symptoms was set as 10 .

\section{Covariates}

Data on chronic diseases were collected based on patients' self-report of having one or more of the following four major chronic diseases diagnosed by a doctor: hypertension, diabetes, arthritis, and dementia. Potential covariates of medical care utilization among elders in South Korean population were age, sex, marital status, education, household income, health insurance, living location, active daily living limitation, physical activity, smoking, alcohol consumption, and obesity [18].

\section{Analysis}

First of all, we analyzed the general characteristics of the participants using a $\chi^{2}$ test. Second, we estimated the relationship between suffering depressive symptoms and medical care utilization using logistic regression analysis. Third, we analyzed subgroups by independent variables, such as each chronic disease. All statistical analyses were conducted using SAS 9.4. 
Since the KLoSA data is fully blinded of specified individual information and has been released to the public for scientific or research use, ethical approval was not required for this study.

\section{Results}

The sample included 3,921 South Korean older adults with chronic diseases who were aged 55 years or older in 2018 (Table 1). Among the participants, 961 (32.5\%) had experienced depressive symptoms and $2,960(67.5 \%)$ did not. In the depressive symptoms group, 180 (18.7\%) patients underwent inpatient hospitalization, and 529 (55.0\%) visited an outpatient clinic.

The results of the multiple regression analysis provided estimated odds ratios (ORs) of health care utilization (Table 2). Compared to patients not suffering from depressive symptoms, those with depressive symptoms had fewer outpatient visits $(\mathrm{OR}=0.81,95 \%$ confidence interval $[\mathrm{Cl}]=0.67-0.98)$ and more inpatient hospitalizations $(\mathrm{OR}=1.49,95 \% \mathrm{Cl}=1.13-1.97)$.

According to the subgroup analysis by covariates, men with depressive symptoms visited outpatient facilities less $(\mathrm{OR}=0.69,95 \% \mathrm{Cl}=0.48-0.97)$ and had more inpatient hospitalizations $(\mathrm{OR}=1.81,95 \% \mathrm{Cl}$ $=1.04-3.16$ ) than did those with sound mental health (Table 3). In contrast, women did not show significant differences. Older adults with depressive symptoms and limited activities of daily living (ADL) experienced more inpatient hospitalizations $(\mathrm{OR}=2.24,95 \% \mathrm{Cl}=1.59-3.15)$ compared to those without limited $A D L(O R=1.45,95 \% \mathrm{Cl}=1.06-1.97)$.

Figures $1 \mathrm{a}$ and $1 \mathrm{~b}$ show the subgroup analyses according to the groups of each chronic diseases such as hypertension, diabetes, joint disease, and combinations of chronic diseases. Odds ratio of elders with hypertension and diabetes was $(\mathrm{OR}=2.77,95 \% \mathrm{Cl}=1.45-5.30)$ in hospitalizations, although, the odds ratio of visiting outpatient was decreased but no statistical significance, patient with diabetes showed $(\mathrm{OR}=0.51,95 \% \mathrm{Cl}=0.24-1.09)$.

\section{Discussion}

This study aimed to examine the association between depressive symptoms, SESs, and medical care utilization in a South Korean older adult population. Our results indicate that there are statistically meaningful differences in medical care utilization among elderly individuals with chronic diseases. Compared to patients with good mental health, patients with depressive symptoms had a higher frequency of inpatient hospitalization $(\mathrm{OR}=1.49,95 \% \mathrm{Cl}=1.13-1.97)$. This result represents two effects of depressive symptoms on health. First, depressive symptoms negatively affected health condition. In previous studies, depressive symptoms were associated with increased vascular disease and risk factors for vascular disease development [2]. Many studies have stated that suffering depressive symptoms is related with a meaningful increase in the chance of coronary heart diseases [19]. Another study revealed that the chance of developing metabolic syndrome was about 2.5 times higher that observed patients with depressive symptoms [20]. One of the systematic review of some longitudinal studies stated that 
patient with depressive symptoms were at a higher risk of metabolic disease relative to people without depressive symptoms [21,22]. Moreover, one of a large population study found that older adults with depressive symptoms showed meaningfully increased risk of diagnosed chronic diseases such as hypertension, after adjusting for socioeconomic and demographic variables and comorbid chronic diseases, than did those without depressive symptoms [23]. Second, patients with depressive symptoms may undergo worsening of chronic diseases [12]. In this study, patients with depressive symptoms visited outpatient clinics less frequently than did those without depressive symptoms. This may disrupt the continuity of disease care and worsen disease conditions [24]. Therefore, patients with depressive symptoms tend to be hospitalized more often than those without depressive symptoms.

There were some significant findings from the analysis of subgroups according to independent variables. First, compared with men without depressive symptoms, those with depressive symptoms usually had fewer outpatient visits and were hospitalized more. However, women did not show this pattern. Men with depressive symptoms tend to neglect their own chronic disease, which results in worsening of condition and hospitalization. Second, in the experience of inpatient hospitalization, those covered by the $\mathrm{NHI}$ experienced more inpatient hospitalizations than those supported by medical aid with depressive symptoms. This result may imply a health care expenditure issue. Although medical aid covers almost of hospital inpatient fees, patients with depressive symptoms who had low property or earned low income could not be capable of expenditure for inpatient hospitalization except for fees. Therefore, they had fewer inpatient hospitalizations than that of the patients covered by NHI. Regarding other variables, such as $A D L$, patients with depressive symptoms and $A D L$ limitation experienced more inpatient hospitalizations than did those without ADL limitation. This result implies that limited ADL may affect patients with depressive symptoms more than it does mentally healthy patients. A previous study also showed that ADL limitation was associated with hospitalization [25]. In South Korea, approximately 500 thousand patients were hospitalized in nursing hospitals in 2019 , and it is difficult to estimate how many patients used nursing home facilities because of their inability to perform daily activities [26]. Therefore, limitation of ADL may lead to increased inpatient hospitalization and health care burden.

In the analysis of each chronic disease type, the risk of hospitalization seemed to increase with each chronic disease. Patients with chronic disease showed an increased OR for inpatient hospitalization, although the difference was not statistically significant. However, patients with hypertension and diabetes showed significantly increased values $(\mathrm{OR}=2.77,95 \% \mathrm{Cl}=1.455 .30)$. On the other hand, these patients showed a relatively lower OR for outpatient visits, but this was not statistically significant. These results imply that depressive symptoms may negatively affect primary care for chronic diseases, especially hypertension and diabetes. Depressive symptoms results in decreased primary care visits and may worsen chronic diseases [27]. This may cause an increase in inpatient hospitalization [22].

Unfortunately, there were some limitations of this study. First, as this was a cross-sectional study, causality could not be established. Therefore, the relationships found in this study should be confirmed using longitudinal studies or other research designs. Second, this study might have recall bias issue as we used self-stated information for variables. This study may have had a selection bias because of the 
potential reluctance of participants in responding honestly regarding their depressive symptoms and health status. Third, this study did not show any differences based on sex, which was simply included as a part of the independent variables.

Despite these limitations, the study has some advantages in terms of its nationally representative large sample size and handling of the aspects relevant to medical care utilization, especially compared to former studies. A lot of studies have examined the associations between health status of older adults and their medical care usage $[2,22,27]$. This study, however, considered not only depressive symptoms but also took into account common chronic diseases, such as diabetes and hypertension, which may affect the medical care use of this population. Unfortunately, in South Korea, there is insufficient governmental support or incentives for patients who suffer from depressive symptoms. There are policies aimed at people suffering from chronic diseases or depressive symptoms. However, we did not find any policies that targeted patients with both depressive symptoms and chronic diseases. Our results showed that depressive symptoms may be related to chronic disease management, and the government needs to pay close attention to older adults with such complex health conditions.

\section{Conclusions}

This study, which used a sample of elders from South Korean population, showed that depressive symptoms may result in a decrease in outpatient visits and an increase in inpatient hospitalization because of chronic diseases. Therefore, health care workers should be encouraged to effectively treat older adults with depressive symptoms in primary clinics, which may lead to a decrease in inpatient hospitalization. Although this relationship is still not to be supported by causal studies, our results support that depressive symptoms are an important part of chronic disease management and, therefore, should be considered when drafting health care policies.

\section{Declarations}

\section{Availability of data and materials}

This study used data from the Korean Longitudinal Study of Aging (KLoSA), which was the primary source of data for our study. DATA from https://survey.keis.or.kr/eng/index.jsp

\section{Consent for publication}

Not applicable.

\section{Competing interests}

The authors declare no conflicts of interest associated with the materials presented in this paper.

\section{Funding}


There was no funding resources of this paper

\section{Acknowledgements}

We are grateful to the KEIS for providing data from the Korean Longitudinal Study of Aging (KLoSA), which was the primary source of data for our study.

\section{Authors' contributions}

YSP analyzed and interpreted the data and was a major contributor in writing the manuscript. ECP was edited and reviewed paper. All authors read and approved the final manuscript

\section{References}

1. OECD. Society at a Glance $2019 ; 2019$

2. Alexopoulos GS. Depression in the elderly. The Lancet. 2005;365(9475):1961-1970.

3. Beekman AT, Deeg DJ, Braam AW, Smit JH, Van Tilburg W. Consequences of major and minor depression in later life: a study of disability, well-being and service utilization. Psychol Med. 1997;27(6):1397-1409.

4. Wong SY, Mercer SM, Leung J, Woo J. The relationship between clinically relevant depressive symptoms and episodes and duration of all cause hospitalization in Southern Chinese elderly. $J$ Affect Disord. 2009;113(3):272-278.

5. McCabe MP, Davison T, Mellor D, George K. Barriers to care for depressed older people: perceptions of aged care among medical professionals. Int J Aging Hum Dev. 2009;68(1):53-64.

6. Luber MP, Meyers BS, Williams-Russo PG, Hollenberg JP, DiDomenico TN, Charlson ME, et al. Depression and service utilization in elderly primary care patients. Am J Geriatr Psychiatry. 2001;9(2):169-176.

7. Katon WJ, Lin E, Russo J, Unutzer J. Increased medical costs of a population-based sample of depressed elderly patients. Arch Gen Psychiatry. 2003;60(9):897-903.

8. Himelhoch S, Weller WE, Wu AW, Anderson GF, Cooper LA. Chronic medical illness, depression, and use of acute medical services among Medicare beneficiaries. Med Care. 2004;42(6):512-521.

9. Korean Statistical Information Service. Statistical Database: Population - Vital status (indicator) [cited 2020 Jun 30]. Available from: https://kosis.kr/statHtml/statHtml.do? orgld=117\&tblld=DT_117071_018\&conn_path=I2.

10. Friedman B, Delavan RL, Sheeran TH, Bruce ML. The effect of major and minor depression on Medicare home healthcare services use. J Am Geriatr Soc. 2009;57(4):669-675.

11. Stein MB, Cox BJ, Afifi TO, Belik SL, Sareen J. Does co-morbid depressive illness magnify the impact of chronic physical illness? A population-based perspective. Psychol Med. 2006;36(5):587-596.

12. Hopman P, Heins MJ, Korevaar JC, Rijken M, Schellevis FG. Health care utilization of patients with multiple chronic diseases in the Netherlands: Differences and underlying factors. European Journal 
of Internal Medicine. 2016;35:44-50.

13. Jang Y, Kim G, Hansen L, Chiriboga DA. Attitudes of older Korean Americans toward mental health services. Journal of the American Geriatrics Society. 2007;55(4):616-620.

14. World Health Organization. Regional Office for the Western P. Republic of Korea health system review: Manila: WHO Regional Office for the Western Pacific; 2015

15. Da Silva RB, Contandriopoulos A-P, Pineault R, Tousignant P. A global approach to evaluation of health services utilization: concepts and measures. Healthcare policy = Politiques de sante. 2011;6(4):e106-e117.

16. National Academies of Sciences, Engineering, and Medicine; Health and Medicine Division; Board on Health Care Services; Committee on Health Care Utilization and Adults with Disabilities. Health-Care Utilization as a Proxy in Disability Determination. Washington (DC): National Academies Press (US); 2018 Mar 1. 2, Factors That Affect Health-Care Utilization.

17. Kim DB, Sohn ES. A Meta- analysis of the Variables Related to Depression in Elderly. Journal of the Korea Gerontological Society. 2005;25(4):167-187.

18. Won CW, Rho YG, Kim SY, Cho BR, Lee YS. The Validity and Reliability of Korean Activities of Daily Living(K-ADL) Scale. J Korean Geriatr Soc. 2002;6(2):98-106.

19. Gallagher D, O'Regan C, Savva GM, Cronin H, Lawlor BA, Kenny RA. Depression, anxiety and cardiovascular disease: which symptoms are associated with increased risk in community dwelling older adults? J Affect Disord. 2012;142(1-3):132-138.

20. Maatouk I, Herzog W, Böhlen F, Quinzler R, Löwe B, Saum KU, et al. Association of hypertension with depression and generalized anxiety symptoms in a large population-based sample of older adults. $\mathrm{J}$ Hypertens. 2016;34(9):1711-1720.

21. Wu Q, Kling JM. Depression and the Risk of Myocardial Infarction and Coronary Death: A MetaAnalysis of Prospective Cohort Studies. Medicine (Baltimore) 2016;95(6):e2815.

22. Grover A, Joshi A. An overview of chronic disease models: a systematic literature review. Global journal of health science. 2014;7(2):210-227.

23. Blaine B. Does depression cause obesity?: A meta-analysis of longitudinal studies of depression and weight control. J Health Psychol. 2008;13(8):1190-1197.

24. Kim W, Jang SY, Lee TH, Lee JE, Park EC. Association between continuity of care and subsequent hospitalization and mortality in patients with mood disorders: Results from the Korea National Health Insurance cohort. PLOS ONE. 2018;13(11):e0207740.

25. Fuino M, Rudnytskyi I, Wagner J. On the characteristics of reporting ADL limitations and formal LTC usage across Europe. European Actuarial Journal 2020;10(2):557-597.

26. Korean Statistical Information Service. Statistical Database: Population - Vital status (indicator) [cited 2020 Jun 30]. Available from: https://kosis.kr/statHtml/statHtml.do? orgld=117\&tblld=DT_117030_001. 
27. Bower P, Macdonald W, Harkness E, Gask L, Kendrick T, Valderas JM, et al. Multimorbidity, service organization and clinical decision making in primary care: a qualitative study. Family Practice. 2011;28(5):579-587.

\section{Tables}


Table 1. General characteristics of study participants

\section{Variables}

Inpatient

Outpatient

Total

Total

\section{Depression}

Yes

No

\section{Age (years)}

$55-64$

65-74

$75+$

Sex

Female

Male

\section{Marital status}

Unmarried

Married

\section{Education}

Highschool

Under middleschool

Household income

\begin{tabular}{llllllllll}
$1 \mathrm{Q}$ & 1,014 & 92 & 9.1 & 922 & 90.9 & 554 & 54.6 & 460 & 45.4 \\
\hline $2 \mathrm{Q}$ & 1,032 & 125 & 12.1 & 907 & 87.9 & 566 & 54.8 & 466 & 45.2 \\
\hline $3 \mathrm{Q}$ & 942 & 124 & 13.2 & 818 & 86.8 & 566 & 60.1 & 376 & 39.9 \\
\hline $4 \mathrm{Q}$ & 933 & 124 & 13.3 & 809 & 86.7 & 557 & 59.7 & 376 & 40.3
\end{tabular}

\section{Insurance}

National Health Insurance $(\mathrm{NHI})$

$\begin{array}{lllllllll}961 & 180 & 18.7 & 781 & 81.3 & 529 & 55.0 & 432 & 45.0 \\ 2,960 & 285 & 9.6 & 2,675 & 90.4 & 1,714 & 57.9 & 1,246 & 42.1\end{array}$

\begin{tabular}{lllllllll}
868 & 61 & 7.0 & 807 & 93.0 & 443 & 51.0 & 425 & 49.0 \\
\hline 1,313 & 162 & 12.3 & 1,151 & 87.7 & 740 & 56.4 & 573 & 43.6 \\
\hline 1,740 & 242 & 13.9 & 1,498 & 86.1 & 1,060 & 60.9 & 680 & 39.1
\end{tabular}

$\begin{array}{lllllllll}2,428 & 303 & 12.5 & 2,125 & 87.5 & 1,394 & 57.4 & 1,034 & 42.6\end{array}$

$\begin{array}{lllllllll}1,493 & 162 & 10.9 & 1,331 & 89.1 & 849 & 56.9 & 644 & 43.1\end{array}$

$\begin{array}{lllllllll}1,184 & 169 & 14.3 & 1,015 & 85.7 & 680 & 57.4 & 504 & 42.6\end{array}$

$\begin{array}{lllllllll}2,737 & 296 & 10.8 & 2,441 & 89.2 & 1,563 & 57.1 & 1,174 & 42.9\end{array}$

$\begin{array}{lllllllll}1,303 & 121 & 9.3 & 1,182 & 90.7 & 700 & 53.7 & 603 & 46.3\end{array}$ $\begin{array}{lllllllll}2,618 & 344 & 13.1 & 2,274 & 86.9 & 1,543 & 58.9 & 1,075 & 41.1\end{array}$ 
Medical Aid Program

$$
208
$$

32

$15.4 \quad 176$

$84.6 \quad 128$

$61.5 \quad 80$

38.5

\section{Location}

Metropolitan

1,593

$1,593 \quad 177$

Urban

$1,309 \quad 161$

11.1

$1,019 \quad 127$

$12.3 \quad 1,148$

Rural

12.5

$\begin{array}{lllllllll}3,672 & 367 & 10.0 & 3,305 & 90.0 & 2,111 & 57.5 & 1,561 & 42.5 \\ 249 & 98 & 39.4 & 151 & 60.6 & 132 & 53.0 & 117 & 47.0\end{array}$

\section{Private health insurance}

\begin{tabular}{llllllllll} 
Yes & 923 & 91 & 9.9 & 832 & 90.1 & 544 & 58.9 & 379 & 41.1 \\
\hline No & 2,998 & 374 & 12.5 & 2,624 & 87.5 & 1,699 & 56.7 & 1,299 & 43.3
\end{tabular}

\section{Physical activity}

\begin{tabular}{llllllllll}
\hline Yes & 1,246 & 134 & 10.8 & 1,112 & 89.2 & 756 & 60.7 & 490 & 39.3 \\
\hline No & 2,675 & 331 & 12.4 & 2,344 & 87.6 & 1,487 & 55.6 & 1,188 & 44.4
\end{tabular}

\section{Smoke}

Neversmoker

Ex-smoker

Current smoke

\section{Drink alcohol}

Yes

No

\section{Obesity}

Yes

No

No. of chronic diseases

\begin{tabular}{llllllllll}
1 & 2,206 & 192 & 8.7 & 2,014 & 91.3 & 1,165 & 52.8 & 1,041 & 47.2 \\
\hline 2 & 1,324 & 198 & 15.0 & 1,126 & 85.0 & 829 & 62.6 & 495 & 37.4 \\
\hline $3-$ & 391 & 75 & 19.2 & 316 & 80.8 & 249 & 63.7 & 142 & 36.3 \\
\hline
\end{tabular}


Due to technical limitations, Table 2 and 3 are only available as a download in the Supplemental Files section.

\section{Figures}
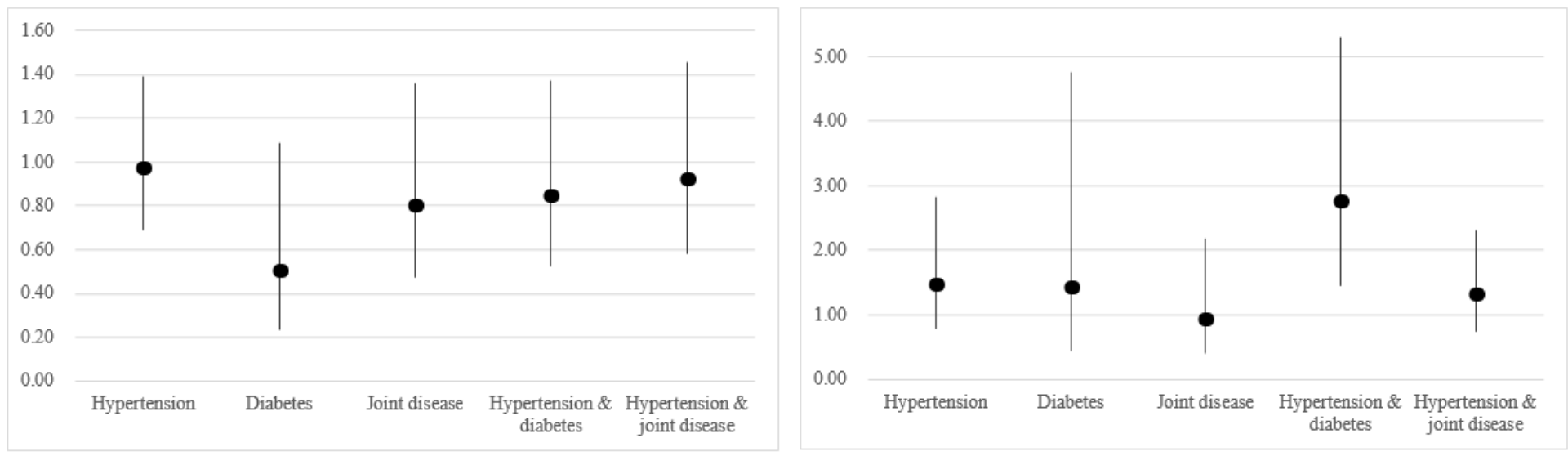

\section{Figure 1}

a. Subgroup analysis of outpatient visit by chronic disease types b. Subgroup analysis of hospitalization by chronic disease types

\section{Supplementary Files}

This is a list of supplementary files associated with this preprint. Click to download.

- Table2and3.docx 